\title{
ON THE EXISTENCE OF SATURATED MODELS OF STABLE THEORIES
}

\author{
VICTOR HARNIK
}

ABSTRACT. It is proven that a theory $T$ stable in a power $\lambda$, $\lambda>|T|$, has a saturated model of cardinality $\lambda$.

The purpose of this note is to show that a theory stable in the power $\lambda$, $\lambda>|T|$, has a saturated model of cardinality $\lambda$. This statement has been well known and easily seen for regular $\lambda$ (even for $\lambda=|T|$ ). We shall, therefore, consider the case of a singular $\lambda>|T|$. Our result was stated in [1] where we gave a proof for the particular case of totally transcendental theories.

Our method of proof was inspired by Shelah's Theorem 4.3 in [8]. It should be mentioned that Shelah extended our result to the case of a singular $\lambda=|T|$ (a proof will appear in [9]).

A proof of a somewhat weaker version of our result is presented in [0] and uses a notion of rank for types.

Before proceeding to the proof itself, we review, in $\$ 1$, the main results about stable theories.

0 . Notations and terminology. Our notations will be standard. $L$ will be a first order finitary language and $T$ a complete $L$-theory. We denote structures by $\mathfrak{X}, \mathfrak{B}, \mathfrak{U}_{0}$, etc. and their respective universes by $A, B, A_{0}$, etc. We follow Shelah [6], [7] and assume the existence of a huge model $B_{0} \vDash T$ such that every other model of $T$ which comes into consideration is an elementary substructure of $B_{0}$. $\vDash \psi\left(a_{0}, \ldots, a_{n-1}\right)$ will mean that $\psi\left(v_{0}, \ldots, v_{n-1}\right)$ is satisfied by $a_{0}, \ldots, a_{n-1}$ in $B_{0} . C, D$ will denote subsets of $B_{0} . L(C)$ will be the language obtained from $L$ by adding individual constants as names for the elements of $C$. We shall not distinguish between an element and its name. $p(a, C)$ will denote the type of $a$ over $C$ i.e., $p(a, C)=\left\{\psi\left(v_{0}\right): \psi\left(v_{0}\right) \in L(C)\right.$, $\vDash \psi(a)\} . S(C)$ will be the set of all types of elements over $C$. For $q \in S(C)$, $C_{1} \subset C, q \mid C_{1}=q \cap L\left(C_{1}\right)$ will be the reduct of $q$ to $C_{1}$.

A model $\mathscr{C} \vDash T$ is called $\lambda$-saturated if for all $C \subset A$ with $|C|<\lambda$, every type $p \in S(C)$ is realized by an element of $A$. 2 is saturated if it is $|A|$-saturated.

$(X,<)$, where $<$ is a linear order, is an ordered set of indiscernibles over $D$ if, for any $n<\omega$, any two increasing $n$-tuples of elements of $X$

Received by the editors April 29, 1974 and, in revised form, August 12, 1974. AMS (MOS) subject classifications (1970). Primary 02B10, 02H05.

Key words and phrases. Stable theory, saturated model. 
satisfy the same formulas of $L(D) . X$ is a set of indiscernibles over $D$ if any two $n$-tuples of distinct elements of $X$ satisfy the same formulas of $L(D)$. These notions are naturally extended to those of an ordered set, resp. a set, of indiscernible $k$-tuples (cf. e.g. Shelah [6], [7]).

${ }^{\kappa} X\left({ }^{<} X\right)$ will be the set of $\kappa$-sequences (of sequences of length $<\kappa$ ) of elements of $X$. If $\eta \in{ }^{\kappa} X, \alpha<\kappa$, then $\eta \mid \alpha$ will be the initial segment of $\eta$ of length $a$. We shall denote by $\bar{a}, \bar{b}$, etc. finite sequences of elements.

1. Preliminaries. In this section, we review some of the main notions connected with stability.

$T$ is called stable in $\lambda$ if for all $C,|C|=\lambda$ implies that $|S(C)|=\lambda . T$ is called stable if it is stable in some power.

It is easy to see that if $T$ is stable in $\lambda \geq|T|, \lambda$ regular, then $T$ has a safurated model of power $\lambda$. If $\lambda$ is singular, one still gets a $\lambda^{\prime}$-saturated model of power $\lambda$ for every regular $\lambda^{\prime}<\lambda$.

Another property is that if $T$ is stable then every ordered set of indiscernibles in a model of $T$ is a set of indiscernibles (cf. Morley [3] and Ressayre [4]).

Definitions 1.1 and 1.3 below, though very important, are not used in our proof.

Definition 1.1. A Morley tree of height $\mu$ is a family $\left\{\phi_{s}\left(v_{0}, \bar{a}_{s}\right): s \in\right.$ $<\mu_{2}$ such that:

(a) for all $\eta \in \epsilon^{\mu}$ the set of formulas $\Phi_{\eta}=\left\{\phi_{\eta \mid a}\left(v_{0}, \bar{a}_{\eta \mid a}\right): a<\mu\right\}$ is consistent with $T$;

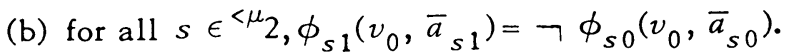

It is immediately seen that if $T$ is stable then $T$ does not have arbitrarily high Morley trees. For $T$ stable let $\mu(T)$ be the first cardinal such that $T$ has no Morley tree of height $\mu(T)$. Less immediate but fairly easy is

Proposition 1.2 (Shelah [5]). If $T$ is stable then $\mu(T) \leq|T|^{+}$.

A more involved notion is

Definition 1.3. A Shelah tree is a family $\left\{\psi_{s}\left(v_{0}, \bar{a}_{s}\right): s \epsilon^{<\kappa} \omega\right\}$ of formulas such that:

(a) For every $\eta \in \epsilon^{\kappa} \omega$, the set of formulas $\Psi_{\eta}=\left\{\psi_{\eta \mid a}\left(v_{0}, \bar{a}_{\eta \mid a}\right): a<\kappa\right\}$ is consistent with $T$.

(b) For every $a<\kappa$ there is a formula $\chi_{a}\left(v_{0}, \bar{x}\right)$ and a natural number $n$ (a) such that for any $s \in{ }_{\omega,}^{a} \psi_{s m}\left(v_{0}, \bar{a}_{s m}\right)=\chi_{a}\left(v_{0}, \bar{a}_{s m}\right)$ for all $m<\omega$, and, any subset of $\left\{\psi_{s m}\left(v_{0}, \bar{a}_{s m}\right): m<\omega\right\}$ which has more than $n(\alpha)$ elements is inconsistent.

Remark. Notice that condition 1.3(b) says nothing about the formulas $\psi_{s}\left(v_{0}, \bar{a}_{s}\right)$ for $s$ a sequence whose length is a limit ordinal. A similar remark applies to Definition 1.1 . 
For a stable theory $T$ define $\kappa(T)$ to be the first cardinality such that $T$ has no Shelah tree of height $\kappa(T)$. It is easy to see that $\kappa(T) \leq \mu(T)$ and strict inequality is possible. Thus, $\kappa(T) \leq|T|^{+}$.

We are now going to reproduce the two important notions of splitting of types. Rather than defining "splitting" we define "nonsplitting".

Definition 1.4 (Shelah [9]). (a) A type $p \in S(C)$ does not split over $D \subset$ $C$ if for all $n<\omega$ and for all $\bar{a}, \bar{b} n$-tuples from $C$, if $\bar{a}$ and $\bar{b}$ satisfy the same $L(D)$-formulas then for all $\psi\left(v_{0}, \bar{x}\right) \in L(D), \psi\left(v_{0}, \bar{a}\right) \in p$ iff $\psi\left(v_{0}, \bar{b}\right) \in p$.

(b) A type $p \in S(C)$ does not split strongly over $D \subset C$ if for every infinite set $X \subset C$ of indiscernibles over $D$, for all $\bar{a}, \bar{b} n$-tuples of distinct elements of $X$ and for all $\psi\left(v_{0}, \bar{x}\right) \in L(D), \psi\left(v_{0}, \bar{a}\right) \in p$ iff $\psi\left(v_{0}, \bar{b}\right) \in p$. These notions are related to the previous ones in

Theorem 1.5. Let $T$ be stable. (a) (Shelab [6] and the author [1], independently). If $p \in S(C)$ then there is $D \subset C,|D|<\mu(T)$, such that $p$ does not split over $D$.

(b) (Shelah [6]). If $p \in S(C)$ then there is $D \subset C,|D| \subset \kappa(T)$, such that $p$ does not split strongly over $D$.

Proof. (The references in Shelah [6] are 2.7 for (a) and 4.3 for (b).) The proof of (a) is essentially the same as that of 1.3 in [2]; it is similar to, but simpler than, the proof of (b) which we are going to reproduce (in outline) at the suggestion of the referee.

Assuming that a type $p, p \in S(C)$, does not satisfy the conclusion of (b), we construct a Shelah tree of height $\kappa(T)$. This will contradict the definition of $\kappa(T)$. Our construction will yield not only a Shelah tree $\left\{\psi_{s}\left(v_{0}, \bar{a}_{s}\right)\right.$ : $\left.s \epsilon^{<\kappa(T)} \omega\right\}$, but also elementary maps $f_{s}$ such that for all $\alpha<\kappa(T)$ and all $s \in \stackrel{a}{\omega}$, the following conditions hold:

(i) the domain of $f_{s}$ is $D_{s}^{\prime}=\{a$ : $a$ is a member of some sequence $\left.\bar{a}_{s \mid \beta}, \beta \leq \alpha\right\}$ and the range of $f_{s}$ is a set $D_{\alpha}, D_{\alpha} \subset C$ (thus, the range of $f_{s}$ depends only on the length $\alpha$ of $s$, and not on $s$ itself);

(ii) if $t$ is an initial segment of $s$, then $f_{t} \subset f_{s}$; and

(iii) $\psi_{s}\left(v_{0}, f_{s}\left(\bar{a}_{s}\right)\right) \in p$.

The construction will be done by induction on $\alpha<\kappa(T)$, the induction assumption being that $\psi_{s}\left(v_{0}, \bar{a}_{s}\right)$ and $f_{s}$ have been defined for all $s$ of length $<\alpha$ in such a way that they satisfy conditions 1.3 (b) and (i)-(iii) (notice that conditions (i)-(iii) imply 1.3(a)). The case of a limit is trivial (take $\psi_{s}\left(v_{0}, \bar{a}_{s}\right)$ to be $v_{0}=v_{0}$; see the Remark just after Definition 1.3). To cope with the case of $\alpha=\beta+1$ we must indicate how to define $\psi_{s m}\left(v_{0}, \bar{a}_{s m}\right)$ and $f_{s m}$ for all $m<\omega$ and $s$ of length $\beta$. By the induction assumption, we are given the set $D_{\beta}$ (the common range of the functions $f_{s}, s$ of length $\beta$ ) and it satisfies that $D_{\beta} C C$ and $\left|D_{\beta}\right|<\kappa(T)$. As we assume $1.5(\mathrm{~b})$ to be 
false, $p$ splits strongly over $D_{\beta}$. It follows that there exist a countable infinite set $X=\left\{x_{0}, x_{1}, \ldots\right\} \subset C$ of indiscernibles over $D_{\beta}$, a formula $\delta\left(v_{0}, \bar{u}\right)$ with $\bar{u}$ a $k$-tuple and disjoint $k$-tuples, say, $\bar{y}_{0}=\left\langle x_{0}, \ldots, x_{k-1}\right\rangle$, $\bar{y}_{1}=\left\langle x_{k}, \ldots, \bar{x}_{2 k-1}\right\rangle$ such that $\delta\left(v_{0}, \bar{y}_{0}\right) \wedge \neg \delta\left(v_{0}, \bar{y}_{1}\right) \in p$. Given any $s$ of length $\beta$, let $B=\left\{b_{0}, b_{1}, \ldots\right\}$ be a set such that $D_{s}^{\prime} \cup B$ is elementarily isomorphic to $D_{\beta} \cup X$ by an isomorphism extending $f_{s}$ and mapping $b_{i}$ to $x_{i}, i<\omega$. For $m<\omega$, define $\bar{a}_{s m}^{\prime}=\left\langle b_{2 k m}, \ldots, b_{2 k m+k-1}\right\rangle, \bar{a}_{s m}^{\prime \prime}=$ $\left\langle b_{2 k m+k}, \ldots, b_{2 k m+2 k-1}\right\rangle$ and $\bar{a}_{s m}=\bar{a}_{s m}^{\prime \prime} \bar{a}_{s m}^{\prime \prime}$. Let $\psi_{s m}\left(v_{0}, \bar{a}_{s m}\right)=$ $\delta\left(v_{0}, \bar{a}_{s m}^{\prime}\right) \wedge-1 \delta\left(v_{0}, \bar{a}_{s m}^{\prime \prime}\right)$ and let $f_{s m}$ be an isomorphism extending $f_{s}$ and mapping $\bar{a}_{s m}$ to $\bar{y}_{0} \bar{y}_{1}$. We are now one small but important step short of the complete proof. We must show that condition $1.3(\mathrm{~b})$ holds for $\left\{\psi_{s m}\left(v_{0}, \bar{a}_{s m}\right)\right.$ : $m<\lambda\}$ for all $s$ of length $\beta$. This last step will be illuminative because it will explain how that condition arises naturally.

We have defined above $\bar{y}_{0}$ and $\bar{y}_{1}$. Define in general $\bar{y}_{i}=\left\langle x_{2 i k}, \ldots\right.$, $\left.x_{2 i k+k-1}\right\rangle$. Then $\left\{\bar{y}_{0}, \bar{y}_{1}, \ldots\right\}$ is a set of indiscernible $k$-tuples which is isomorphic to any of the sets $\left\{\bar{a}_{s 0}^{\prime}, \bar{a}_{s 0}^{\prime \prime}, \bar{a}_{s 1}^{\prime}, \bar{a}_{s 1}^{\prime \prime}, \ldots\right\}$ for $s$ of length $\beta .1 .3(\mathrm{~b})$ will follow from

Claim (Shelah [7, 5.9A]). There is an $n<\omega$ such that any subset of $\left\{\delta\left(v_{0}, \bar{y}_{2 i}\right) \wedge \neg \delta\left(v_{0}, \bar{y}_{2 i+1}\right): i<\omega\right\}$ having cardinality $>n$ is inconsistent.

Proof of the claim. Extend $\left\{\bar{y}_{i}: i<\omega\right\}$ to a set $\left\{\bar{y}_{i}: i<\lambda\right\}$ of indiscernible $k$-tuples, where $\lambda$ is a cardinality in which $T$ is stable. If the claim is false, then an easy compactness argument shows that for every $I \subset \lambda$, the set of formulas $\Delta_{I}=\left\{\delta\left(v_{0}, \bar{y}_{i}\right): i \in I\right\} \cup\left\{\neg \delta\left(v_{0}, \bar{y}_{i}\right): i \notin I\right\}$ is consistent with $T$. This yields $2^{\lambda}$ distinct types over a set of power $\lambda$, contradicting the $\lambda$-stability of $T$.

The proof of $1.5(\mathrm{~b})$ is now complete.

The following is a special case of $1.5(\mathrm{~b})$ :

Corollary 1.6 (Shelah). Let $T$ be stable. If $X$ is a set of indiscernibles over $D$ and $C_{0}$ is a finite set, then there is a set $X_{0} \subset X$ with $\left|X_{0}\right|<$ $\kappa(T)$ such that $X-X_{0}$ is a set of indiscernibles over $D \cup C_{0}$ (in fact, over $\left.D \cup C_{0} \cup X_{0}\right)$.

As a special case of $1.5(\mathrm{a})$, the author independently obtained 1.6 weakened with $\left|X_{0}\right|<\mu(T)$ instead of $\left|X_{0}\right|<\kappa(T)$ (cf. [1] or $[2,1.3]$ ).

We next state a technical lemma which we use in our proof. Arguments of this sort have been repeatedly used; see, e.g., Shelah [5], [6], [7], and go, in essence, back to Morley [3].

Lemma 1.7. Let $q \in S(C), D \subset C$ and let $\left\{a_{\beta}\right\}_{\beta<a}$ be a sequence of elements of $C$ such that for all $\gamma<\alpha, p\left(a_{\gamma}, D \cup\left\{a_{\beta}\right\}_{\beta<\gamma}\right)=q \mid D \cup\left\{a_{\beta}\right\}_{\beta<\gamma}$.

(a) If $q$ does not split over $D$ then $\left\{a_{\beta}\right\}_{\beta<a}$ is a set of indiscernibles over $D$. 
(b) If $q$ does not split strongly over $D$ and if $\left\{a_{\beta}\right\}_{\beta<\omega}$ is a set of indiscermibles over $D$, then so is $\left\{a_{\beta}\right\}_{\beta<a}$.

Proof of (b). It suffices to show that the sequence $\left\{a_{\beta}\right\}_{\beta<\alpha}$ is an ordered set of indiscernibles. We prove this by induction on $\alpha$. For $\alpha=\omega$ there is nothing to prove and for $a$ limit the induction is trivial. Assume that $\alpha=\gamma$ +1 and $\left\{a_{\beta}\right\}_{\beta<\gamma}$ is a set of indiscernibles over $D$. To show the same for $\left\{a_{\beta}\right\}_{\beta<a}$ we have to show that $\bar{a}, a_{\gamma}$ and $\bar{a}^{\prime}, a_{\beta_{n-1}^{\prime}}$ satisfy the same $L(D)$ formulas where $\bar{a}=\left\langle a_{\beta_{0}}, \ldots, a_{\beta_{n-2}}\right\rangle, \bar{a}^{\prime}=\left\langle a_{\beta_{0}^{\prime}}, \ldots, a_{\beta_{n-2}^{\prime}}\right\rangle$ with $\beta_{0}<$ $\ldots<\beta_{n-2}<\gamma$ and $\beta_{0}^{\prime}<\ldots<\beta_{n-1}^{\prime} \leq \gamma$. Taking into consideration all the assumptions, including the induction assumption, we get that for $\psi\left(\bar{x}, v_{0}\right) \epsilon$ $L(D), \vDash \psi\left(\bar{a}, a_{\gamma}\right)$ iff $\psi\left(\bar{a}, v_{0}\right) \in q$ iff $\psi\left(\bar{a}^{\prime}, v_{0}\right) \in q$ iff $\vDash \psi\left(\bar{a}^{\prime}, a_{\beta_{n-1}^{\prime}}\right)$. Q.E.D.

The most important fact concerning stable theories is, perhaps, the following.

Theorem 1.8 (Shelah [6]). If $T$ is stable then there is $\lambda_{0},|T| \leq \lambda_{0} \leq$ ${ }_{2}|T|$, such that for all $\lambda \geq|T|, T$ is stable in $\lambda$ iff $\lambda \geq \lambda_{0}$ and $\lambda^{k(T)}=\lambda$.

Corollary 1.9. If $T$ is stable in $\lambda$ then $\kappa(T) \leq \mathrm{cf} \lambda$.

2. The theorem. We are going to prove

Theorem 2.1. If $T$ is stable in $\lambda, \lambda$ is singular and $\lambda>|T|$, then $T$ has a saturated model of power $\lambda$.

Proof. Let $\lambda=\Sigma_{i<\mathrm{cf} \lambda} \lambda_{i}$ where $\left\{\lambda_{i}\right\}_{i<\mathrm{cf} \lambda}$ is an increasing sequence of regular cardinals. We may assume that $\lambda_{0}>$ cf $\lambda+|T|$. Take an increasing elementary chain $\left\{\mathscr{C}_{i}\right\}_{i<\mathrm{cf} \lambda}$ of models of $T$ such that $\left|\mathscr{U}_{i}\right| \leq \lambda$ and $\mathscr{U}_{i}$ is $\lambda_{i}$-saturated. The model $\mathscr{U}=\bigcup_{i<c f \lambda} \mathfrak{A}$ has power $\lambda$ and we will show that it is saturated.

Let $C \subset A,|C|<\lambda$ and let $p \in S(C)$. We want to prove that $p$ is realized in 2 . Let $q \supseteq p$ be an extension of $p$ to a type $q \in S(A)$. By 1.5(b), there is $D \subset A,|D|<\kappa(T) \leq$ cf $\lambda$ such that $q$ does not split strongly over $D$. Because the power of $D$ is so small, $D \subset A_{i}$ for some $i<\mathrm{cf} \lambda$. W.l.o.g. we may assume that $D \subset A_{0}$. Define a sequence of elements $b_{\beta}, \beta<|T|^{+}$, such that $b_{\beta} \in A_{0}$ and for all $\gamma<|T|^{+}, p\left(b_{\gamma}, D \cup\left\{b_{\beta}\right\}_{\beta<\gamma}\right)=q \mid D \cup\left\{b_{\beta}\right\}_{\beta<\gamma}$. This is possible since $\mathfrak{l}_{0}$ is $\lambda_{0}$-saturated and $\lambda_{0}>|D|+|T|$. By $1.5(\mathrm{a})$ there is an $\alpha_{0}<$ $|T|^{+}$such that $q \mid D \cup\left\{b_{\beta}\right\}_{\beta<|T|^{+}}$does not split over $D \cup\left\{b_{\beta}\right\}_{\beta<a_{0}}$. It follows, by 1.7(a), that $\left\{b_{\beta}\right\}_{a_{0} \leq \beta<|T|^{+}}$is a sequence of indiscernibles over $D$. Renam-

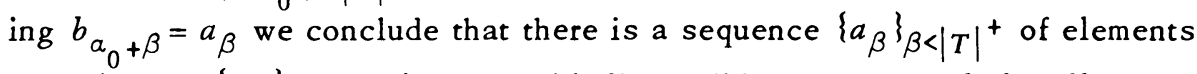
$a_{\beta} \in A_{0}$ s.t. $\left\{a_{\beta}\right\}_{\beta<|T|^{+}}$is a set of indiscernibles over $D$ and, for all $\gamma<$ $|T|^{+}, p\left(a_{\gamma}, D \cup\left\{a_{\beta}\right\}_{\beta<\gamma}\right)=q \mid D \cup\left\{a_{\beta}\right\}_{\beta<\gamma}$. We now come to the heart of the proof. 
Claim 2.2. For every finite set $C_{0}, C_{0} \subset A$, all but less than $|T|^{+}$elements of $\left\{a_{\beta}\right\}_{\beta<|T|^{+}}$satisfy $q \mid C_{0}$.

Proof of 2.2. W.1.o.g. we may assume that $C_{0} \subset A_{2}$. Define $a_{\beta},|T|^{+} \leq$ $\beta<|T|^{+} \cdot 2$, such that $a_{\beta} \in A_{2}$ and for all $\gamma,|T|^{+} \leq \gamma<|T|^{+} \cdot 2$, $p\left(a_{\gamma}, D \cup C_{0} \cup\left\{a_{\beta}\right\}_{\beta<\gamma}\right)=q \mid D \cup C_{0} \cup\left\{a_{\beta}\right\}_{\beta<\gamma}$. Since $q$ does not split strongly over $D$, we conclude, by $1.7(\mathrm{~b})$, that $\left\{a_{\beta}\right\}_{\beta<|T|^{+} \cdot 2}$ is a set of indiscernibles over $D$. By 1.6, there is $X_{0} \subset\left\{a_{\beta}\right\}_{\beta<|T|^{+} \cdot 2}$ with $\left|X_{0}\right|<|T|^{+}$such that $X=\left\{a_{\beta}\right\}_{\beta<|T|^{+} \cdot 2}-X_{0}$ is a set of indiscernibles over $C_{0}$. X certainly contains some $a_{\beta}$ with $\beta \geq|T|^{+}$and that $a_{\beta}$ realizes $q \mid C_{0}$ (by the very definition of $a_{\beta}$ for $\beta \geq|T|^{+}$). It follows that every element of $X$ realizes $q \mid C_{0}$. This proves 2.2 since $X$ contains all but less than $|T|^{+}$elements of $\left\{a_{\beta}\right\}_{\beta<|T|^{+}}$.

Returning to the proof of 2.1, define $\left\{a_{\beta}\right\}|T|^{+} \leq \beta<\lambda$ such that $a_{\beta} \in A$ and for all $\gamma<\lambda, p\left(a_{\gamma}, D \cup\left\{a_{\beta}\right\}_{\beta<\gamma}\right)=q \mid D \cup\left\{a_{\beta}\right\}_{\beta<\gamma}$. This can be done inductively provided one makes sure that $\left\{a_{a}\right\}_{a<\lambda_{i}} \subset A_{i}$ for all $i<\mathrm{cf} \lambda$. Again by 1.7(b), we conclude that $Y=\left\{a_{\beta}\right\}_{\beta<\lambda}$ is a set of indiscernibles over $D$. Also, it follows from 2.2 that for all finite $C_{0} \subset A$ there is $Y_{C_{0}} \subset Y,\left|Y_{C_{0}}\right|<|T|^{+}$ such that every element of $Y-Y_{C_{0}}$ realizes $q \mid C_{0}$.

Let us go back to our initial $C$ with $|C| \subset \lambda$. As $Y^{\prime}=\bigcup\left\{Y_{C_{0}}: C_{0} \subset\right.$ $C, C_{0}$ finite $\}$ has power $\leq|C| \cdot|T|<\lambda$, it follows that the set $Y^{0}-Y^{\prime}$ is nonvoid and every element in it realizes $q \mid C=p$. Q.E.D.

Shelah noticed [7, B3] that our proof shows in fact that whenever $\left\{\mathscr{X}_{i}\right\}_{i<\delta}$ is an increasing elementary chain such that $\mathscr{U}_{i}$ is $\lambda_{i}$-saturated where cf $\delta \geq$ $\kappa(T)$ and $\left\{\lambda_{i}\right\}_{i<\delta}$ is an increasing (not necessarily strictly) sequence of cardinals with $\Sigma_{i<\delta} \lambda_{i}=\lambda>|T|$ then $\mathcal{A}=\bigcup_{i<\delta^{2}}{ }_{i}$ is $\lambda$-saturated. He further noticed that this implies, even for singular $\lambda$, the existence of a $\lambda$-atomic (cf. [2]), $\lambda$-prime model over every $C$ provided that $\lambda>|T|$ and cf $\lambda \geq \kappa(T)$. To construct such a model, one takes an increasing sequence $\left\{\lambda_{i}: i<\operatorname{cf} \lambda\right\}$ of regular cardinals $>|T|$ whose limit is $\lambda$ and then constructs a model $\mathscr{U}$ such that $A=C \cup\left\{a_{a}\right\}_{a<\lambda}$, for all $i<\operatorname{cf} \lambda, A_{i}=C \cup\left\{a_{\alpha}\right\}_{a<\lambda_{i}}$ is the universe of a $\lambda_{i}$-saturated model, and for all $\alpha<\lambda_{i}, p\left(a_{\alpha}, C \cup\left\{a_{\beta}\right\}_{\beta<a}\right)$ is $\lambda_{i}$-isolated (see [2], [4] or [5] for details of such a construction).

\section{REFERENCES}

0. J. T. Baldwin and A. Blass, An axiomatic approach to rank in model theory Ann. Math. Logic 7 (1974), 295-324.

1. V. Harnik, Stable theories and related concepts, Ph.D. Thesis, Hebrew University, 1971.

2. V. Harnik and J.-P. Ressayre, Prime extensions and categoricity in power, Israel J. Math. 10 (1971), 172-185. MR $46 \# 45$.

3. M. D. Morley, Categoricity in power, Trans. Amer. Math. Soc. 114 (1965), 514-538. MR $31 \# 58$.

4. J. P. Ressayre, Sur les théories du premier ordre catégoriques en un cardinal, Trans. Amer. Math. Soc. 142 (1969), 481-505. MR 40 \#32.

5. S. Shelah, Stable theories, Israel J. Math. 7 (1969), 187-202. MR 40 \#7102. 
6.S. Shelah, Finite diagrams stable in power, Ann. Math. Logic $2(1970 / 71)$, no. 1, 69-118. MR $44 \# 2593$.

7. - Stability, the f.c.p. and superstability; model theoretic propreties of formulas in first order theory, Ann. Math. Logic 3 (1971), no. 3, 271-362. MR 47 \#6475.

8. - Saturation of ultrapowers and Keisler's order, Ann. Math. Logic 4 (1972), 75-114. MR 45\#3187.

9. ——, North-Holland, Amsterdam (to appear).

DEPARTMENT OF MATHEMATICS, DARTMOUTH COLLEGE, HANOVER, NEW HAMP. SHIRE 03755

Current address: Department of Mathematics, University of Haifa, Mount Carmel, Haifa, Israel 